\title{
Bogoliubov transformations and exact isolated solutions for simple nonadiabatic Hamiltonians
}

\author{
C. Emary ${ }^{\text {a) }}$ and R. F. Bishop \\ Department of Physics, University of Manchester Institute of Science and Technology \\ (UMIST), P.O. Box 88, Manchester M60 1QD, United Kingdom
}

(Received 10 December 2001; accepted for publication 25 April 2002)

\begin{abstract}
We present a new method for finding isolated exact solutions of a class of nonadiabatic Hamiltonians of relevance to quantum optics and allied areas. Central to our approach is the use of Bogoliubov transformations of the bosonic fields in the models. We demonstrate the simplicity and efficiency of this method by applying it to the Rabi Hamiltonian. (C) 2002 American Institute of Physics.
\end{abstract}

[DOI: 10.1063/1.1490406]

\section{INTRODUCTION}

There exists a class of simple, nonintegrable, nonadiabatic Hamiltonians of the type that find application as models of light-matter interactions, for which it is possible to find exact isolated solutions. Generally these models involve some atomic system, typically characterized by a simple two-level (or multilevel) system, interacting with a number of bosonic fields. Making the familiar rotating-wave approximation usually renders these models completely soluble, but avoiding this approximation maintains the nonintegrability of the models, and gives rise to the possibility of isolated exact solutions. This was first demonstrated for the Jahn-Teller model by Judd, ${ }^{1}$ and these solutions are often referred to as Juddian solutions. Probably the simplest model for which these solutions have been found is the Rabi Hamiltonian ( $\mathrm{RH}$ ), which describes a two-level atom interacting with a single-mode bosonic field via a dipole interaction. ${ }^{2}$ The Juddian solutions of the RH were first discovered by Reik and co-workers, ${ }^{3}$ where they were seen to occur at the level crossings in the energy schema of the system. This turns out to be a general and important feature of these solutions.

Apart from being of interest for what they tell us about the structure and symmetries of these models, the Juddian solutions are of considerable further value. Simple quantum optics and related models, such as the RH, have long been utilized as test cases for various calculational techniques, ${ }^{4-6}$ and the possession of exact solutions facilitates their accurate assessment. Furthermore, the existence of isolated exact solutions in nonintegrable quantum models is also of interest from the perspective of studying possible quantum chaos in such systems. ${ }^{7,8}$ In addition, it is hoped that these exact solutions may serve as useful starting points for perturbative treatments of the entire spectra of these models.

In this paper we present a new and more general method for finding these isolated exact solutions, which we believe to have several advantages over the methods hitherto employed. Judd and Reik, working in the Bargmann representation, have used power series and Neumann series ansätze for the field mode. Neither of these approaches is particularly intuitive and the resulting algebra can become complicated. Kuś and Lewenstein ${ }^{9}$ have given a more concise approach which, as we describe later, is clearly related to the method that we describe here. For models such as the RH they used Bargmann representation ansätze for the field consisting of a finite number of bosonic excitations on top of a coherent state. They have also extended their method to some further systems, such as a three-level system and an auto-ionizing ion.

We believe that the method we outline in this paper is both more intuitive and more efficient

${ }^{a)}$ Electronic mail: emary@dirac.phy.umist.ac.uk 
than those discussed previously, and that it reflects the essential physics of the systems to a greater degree. At the heart of the method is a simple canonical transformation of the bosonic field operators of the models. This transformation suggests the existence of exact solutions in a most direct manner. Our method also has the advantage that it is easy to generalize, and is readily able to be extended to "two-photon" type interactions, in which two photons are required to induce an atomic transition. ${ }^{10}$

The remainder of this paper is organized as follows. In Sec. II we outline our method for finding the Juddian solutions. We describe in some detail the theory of Bogoliubov transformations of a boson mode and pay particular attention to their relation to the coherent and squeezed states. We then use these transformations to investigate the displaced and squeezed harmonic oscillators, to develop insight into the reasoning behind this approach. In Sec. III we apply this method to the Rabi Hamiltonian, as an example of the use of this method. We then finish with some conclusions and indications of further work.

\section{METHODOLOGY}

The models that we consider here consist of an atomic system interacting with one or more bosonic modes. Each of these modes is described by annihilation and creation operators, $b$ and $b^{\dagger}$, respectively, which obey the usual commutation relation,

$$
\left[b, b^{\dagger}\right]=1 .
$$

In general the atomic system will be described in terms of a set of matrices. For example, the two-level system in the RH is described by the SU(2) Pauli matrices.

Our method for finding exact isolated solutions for such systems involves two components. First, one must choose an appropriate representation for the atomic matrices and then, crucially, one performs a Bogoliubov transformation of the operators of the field mode. The nature of this transformation depends upon the type of interaction being considered and, with the correct choice of parameters, it leaves the Schrödinger equation in a form that admits exact solutions with very simple ansätze.

\section{A. Bogoliubov transformations}

A Bogoliubov transformation is a transformation from one description of a field mode in terms of the bosonic operators, $b$ and $b^{\dagger}$, to a description in terms of new bosonic operators, $\widetilde{b}$ and $\widetilde{b}^{\dagger}$, say. This transformation is canonical so that the new operators obey the same commutation relation as the old ones, namely

$$
\left[\widetilde{b}, \widetilde{b}^{\dagger}\right]=1 .
$$

The most general linear Bogoliubov transformation may be viewed as a rotation plus translation of the original oscillator Hilbert space to the new oscillator space,

$$
\begin{gathered}
\tilde{b}=e^{-i \beta}\left(1-|\sigma|^{2}\right)^{-1 / 2}\left(b-\sigma b^{\dagger}-z\right), \\
\tilde{b}^{\dagger}=e^{i \beta}\left(1-|\sigma|^{2}\right)^{-1 / 2}\left(b^{\dagger}-\sigma^{*} b-z^{*}\right),
\end{gathered}
$$

where $\sigma$ and $z$ are complex numbers describing the amplitudes of the rotation and translation, respectively. $\beta$ is a simple, and usually rather unimportant, phase factor. From the outset it is important to note the restriction $|\sigma|<1$ in order to preserve the unitarity of the transformation. In the following we consider two specializations of this transformation, namely a pure translation and a pure rotation. These transformations may be very simply related to the familiar coherent and squeezed states of quantum optics and it is from this standpoint that we introduce the transformations. 


\section{B. Coherent bosons}

The usual Glauber coherent states, $|z\rangle$, may be defined as eigenkets of the single-mode bosonic annihilation operator, ${ }^{11}$

$$
b|z\rangle=z|z\rangle,
$$

where $z$ is a complex number. Such states are readily constructed as the following equivalent forms:

$$
\begin{aligned}
|z\rangle & =e^{-1 / 2|z|^{2}} e^{z b^{\dagger}}|0\rangle \\
& =e^{\left(z b^{\dagger}-z^{*} b\right)}|0\rangle,
\end{aligned}
$$

where we have normalized the coherent state such that $\langle z \mid z\rangle=1$. The exponential operator in Eq. (6) is denoted as follows:

$$
D(z) \equiv e^{\left(z b^{\dagger}-z^{*} b\right)},
$$

and is called the displacement operator. It is a unitary operator and we may readily use it to perform a unitary transformation of the field operators,

$$
\begin{gathered}
D(z) b D^{\dagger}(z)=b-z \equiv a, \\
D(z) b^{\dagger} D^{\dagger}(z)=b^{\dagger}-z^{*} \equiv a^{\dagger} .
\end{gathered}
$$

The operators $D(z)$ form a representation of the Weyl (or Heisenberg-Weyl) group when multiplied by a trivial phase factor $\exp (i \phi)$, with $\phi$ real. The operators $a$ and $a^{\dagger}$ obey the same commutator relation as the original operators, and thus we see this transformation to be a Bogoliubov transformation of the type described earlier as a pure translation. Equations (4) and (8) clearly imply

$$
a|z\rangle=0,
$$

from which we see that the operator $a$ annihilates the coherent state $|z\rangle$. Thus $|z\rangle$ may be considered as the vacuum state of the $a$-type bosons, and we rewrite it accordingly as $|0 ; z\rangle$ $\equiv|z\rangle$,

$$
a|0 ; z\rangle=0 .
$$

We shall call these $a$-type bosons "coherent bosons" and write their number states as $|n ; z\rangle$, such that $a^{\dagger} a|n ; z\rangle=n|n ; z\rangle$.

\section{Displaced harmonic oscillator}

The simplest application of the coherent bosons is to the displaced harmonic oscillator,

$$
H_{D}=\frac{1}{2}(x+\sqrt{2} \lambda)^{2}+\frac{1}{2} p^{2},
$$

in which the center of the oscillator is shifted by an amount $-\sqrt{2} \lambda$. Introducing the harmonic oscillator operators via

$$
\begin{aligned}
& x \equiv \frac{1}{\sqrt{2}}\left(b^{\dagger}+b\right), \\
& p \equiv \frac{i}{\sqrt{2}}\left(b^{\dagger}-b\right),
\end{aligned}
$$


the Hamiltonian reads

$$
H_{D}=b^{\dagger} b+\lambda\left(b^{\dagger}+b\right)+\frac{1}{2}+\lambda^{2} .
$$

By performing a Bogoliubov transformation of the original bosonic operators to a new set of coherent bosons, $a^{\dagger}$ and $a$, such that

$$
a \equiv b+\lambda, \quad a \equiv b^{\dagger}+\lambda,
$$

we may rewrite the Hamiltonian of Eq. (13) in the form

$$
H_{D}=a^{\dagger} a+\frac{1}{2}
$$

The eigenstates of this Hamiltonian are thus clearly seen to be the number states of the $a$-type bosons, with corresponding eigenenergies $E_{n}=n+\frac{1}{2}$.

\section{Squeezed bosons}

Following Bishop and Vourdas ${ }^{12}$ we construct the most general squeezed state, $|z ; \rho, \theta, \beta\rangle$, by acting upon the bosonic vacuum $|0\rangle$ first with the displacement operator $D(z)$ of Eq. (7) and then with the pure squeezing operator $S(\rho, \theta, \beta)$,

$$
|z ; \rho, \theta, \beta\rangle=S(\rho, \theta, \beta) D(z)|0\rangle,
$$

The squeezing operator is given by

$$
S(\rho, \theta, \beta) \equiv \exp \left(-\frac{1}{4} \rho e^{-i \theta} b^{\dagger 2}+\frac{1}{4} \rho e^{i \theta} b^{2}\right) \exp \left(i \beta b^{\dagger} b\right),
$$

where $\rho, \theta, \beta$ are real parameters. It is a unitary operator, $S^{\dagger} S=1$, and provides a representation of the group $\mathrm{SU}(1,1)$. Using a relationship given by Perelomov, ${ }^{13}$ we are able to write the squeezing operator in the equivalent form

$$
S(\sigma, \beta)=\exp \left(\frac{1}{2} \sigma b^{\dagger 2}\right)\left(1-|\sigma|^{2}\right)^{b^{\dagger} b / 2+1 / 4} \exp \left(-\frac{1}{2} \sigma^{*} b^{2}\right) \exp \left(i \beta b^{\dagger} b\right),
$$

where $\beta$ is the same real parameter as above, and $\sigma$ is a complex number with modulus $|\sigma|<1$, given by $\sigma \equiv-e^{-i \theta} \tanh \left(\frac{1}{2} \rho\right)$. Using this expression, we can use the squeezing operator to make unitary transformations of the bosonic annihilation and creation operators,

$$
\begin{gathered}
S(\sigma, \beta) b S^{\dagger}(\sigma, \beta)=e^{-i \beta}\left(1-|\sigma|^{2}\right)^{-1 / 2}\left(b-\sigma b^{\dagger}\right) \equiv c, \\
S(\sigma, \beta) b^{\dagger} S^{\dagger}(\sigma, \beta)=e^{i \beta}\left(1-|\sigma|^{2}\right)^{-1 / 2}\left(b^{\dagger}-\sigma^{*} b\right) \equiv c^{\dagger} .
\end{gathered}
$$

The operators $c$ and $c^{\dagger}$ satisfy the commutation relation $\left[c, c^{\dagger}\right]=1$ and thus the transformation $b, b^{\dagger} \rightarrow c, c^{\dagger}$ is a Bogoliubov transformation of the rotation type. From Eq. (19), it follows that for any function $f\left(b, b^{\dagger}\right)$,

$$
S f\left(b, b^{\dagger}\right) S^{\dagger}=f\left(c, c^{\dagger}\right) \leftrightarrow S f\left(b, b^{\dagger}\right)=f\left(c, c^{\dagger}\right) S .
$$

Equation (20) implies that $S b=c S$ and hence $|z ; \sigma \beta\rangle \equiv|z ; \rho \theta \beta\rangle$ are eigenstates of the annihilation operator $c$,

$$
c|z ; \sigma \beta\rangle=c S(\sigma, \beta)|z\rangle=S(\sigma, \beta) b|z\rangle=z|z ; \sigma \beta\rangle .
$$

If we consider the squeezed vacuum $S|0\rangle=|0 ; \sigma, \beta\rangle=|0 ; \sigma\rangle$, we see that it is independent of $\beta$ and that

$$
c|0 ; \sigma\rangle=0 .
$$


The number states of the $c$-type bosons are denoted $|n ; \sigma \beta\rangle$, such that $c^{\dagger} c|n ; \sigma \beta\rangle=n|n ; \sigma \beta\rangle$. We call the $c$-type bosons "squeezed" bosons.

\section{E. Squeezed harmonic oscillator}

In position representation the squeezed harmonic oscillator has the form

$$
H_{S}=\frac{1}{2}(1+2 \lambda) x^{2}+\frac{1}{2}(1-2 \lambda) p^{2},
$$

where the real parameter $\lambda$ determines the degree of squeezing, with the restriction that $|\lambda|<\frac{1}{2}$. Translating this into the standard bosonic representation defined by Eq. (12) we have

$$
H_{S}=b^{\dagger} b+\frac{1}{2}+\lambda\left(b^{\dagger 2}+b^{2}\right) .
$$

We introduce squeezed $c$-type bosons defined by

$$
c^{\dagger}=\frac{b^{\dagger}+\sigma b}{\sqrt{1-\sigma^{2}}}, \quad c=\frac{b+\sigma b^{\dagger}}{\sqrt{1-\sigma^{2}}},
$$

and leave $\sigma$ real but undetermined for the moment. Making these substitutions into Eq. (24), we have

$$
H_{S}=\frac{1}{\left(1-\sigma^{2}\right)}\left\{\left[-\sigma+\lambda+\lambda \sigma^{2}\right]\left(c^{2}+c^{\dagger 2}\right)+\left(\sigma^{2}+1-4 \lambda \sigma\right)\left(c^{\dagger} c+\frac{1}{2}\right)\right\} .
$$

We eliminate the first term in this Hamiltonian by choosing

$$
-\sigma+\lambda+\lambda \sigma^{2}=0
$$

giving, as one of the two solutions,

$$
\sigma=\frac{(1-\Omega)}{2 \lambda}, \quad \Omega \equiv \sqrt{1-4 \lambda^{2}} .
$$

With this choice, the Hamiltonian becomes

$$
H_{S}=\left\{c^{\dagger} c+\frac{1}{2}\right\} \Omega .
$$

The eigenstates of this Hamiltonian are clearly the number states of the squeezed $c$-type bosons, with eigenenergies

$$
E_{n}=\left\{n+\frac{1}{2}\right\} \Omega .
$$

We note that the other solution of Eq. (27) with $\sigma=(1+\Omega) / 2 \lambda$ leads to the unphysical oscillator with $H_{S}=-\left(c^{\dagger} c+\frac{1}{2}\right) \Omega$, and since this Hamiltonian does not have square-integrable solutions, we discard it.

\section{APPLICATION TO THE RABI HAMILTONIAN}

The Rabi Hamiltonian (RH) describes a two-level atom interacting with a single mode of quantized electromagnetic radiation via a dipole interaction. ${ }^{2}$ It is usually written in the form

$$
H_{\text {Rabi }}=\frac{1}{2} \omega_{0} \sigma_{z}+\omega b^{\dagger} b+g\left(b^{\dagger}+b\right)\left(\sigma_{+}+\sigma_{-}\right),
$$


where $\omega_{0}$ is the atomic level splitting, $\omega$ is the frequency of the boson mode, and $g$ is the coupling strength of the atom to the field. The two-level atom is described by the Pauli pseudo-spin operators, which satisfy the $\mathrm{SU}(2)$ commutation relations

$$
\left[\sigma_{k}, \sigma_{l}\right]=2 i \varepsilon_{k l m} \sigma_{m},
$$

where $k, l, m \in\{x, y, z\}$ with $k \neq l$ and $\varepsilon_{k l m}$ is the antisymmetric Levi-Civita symbol. We have defined the raising and lowering operators as

$$
\sigma_{+} \equiv \sigma_{x}+i \sigma_{y}, \quad \sigma_{-} \equiv \sigma_{x}-i \sigma_{y} .
$$

It is convenient to rescale the Hamiltonian as $H_{\text {Rabi }}=\omega \widetilde{H}_{\text {Rabi }}$, where

$$
\widetilde{H}_{\mathrm{Rabi}}=\widetilde{\omega} \sigma_{z}+b^{\dagger} b+\lambda\left(b^{\dagger}+b\right) \sigma_{x},
$$

and $\widetilde{\omega} \equiv \omega_{0} /(2 \omega)$ and $\lambda \equiv 2 g / \omega$. There is a conserved parity $\Pi$ associated with the Hamiltonian,

$$
\Pi \equiv \exp \left[i \pi\left(b^{\dagger} b+\frac{1}{2}\left(\sigma_{z}+1\right)\right)\right]=-\sigma_{z} \cos \left(\pi b^{\dagger} b\right),
$$

such that $\left[H_{\text {Rabi }}, \Pi\right]=0$. The parity operator $\Pi$ has two eigenvalues, $\pi= \pm 1$. The RH is not known to be integrable, but isolated exact solutions do exist. Here we use the above-outlined technique to find these Juddian solutions.

In order to do this we first require an appropriate matrix representation for the Pauli matrices, which for this model is one in which $\sigma_{x}$ is diagonal. We shall use

$$
\sigma_{x}=\left[\begin{array}{cc}
1 & 0 \\
0 & -1
\end{array}\right], \quad \sigma_{y}=\left[\begin{array}{cc}
0 & i \\
-i & 0
\end{array}\right], \quad \sigma_{z}=\left[\begin{array}{ll}
0 & 1 \\
1 & 0
\end{array}\right] .
$$

In terms of the two-component wave function, $|\Psi\rangle=\left(\begin{array}{c}\left|\Psi_{1}\right\rangle \\ \left|\Psi_{2}\right\rangle\end{array}\right)$, the time-independent Schrödinger equation for the system, $\widetilde{H}_{\text {Rabi }}|\Psi\rangle=E|\Psi\rangle$, then reads

$$
\begin{aligned}
& \widetilde{\omega}\left|\Psi_{2}\right\rangle+\left(b^{\dagger} b+\lambda\left(b^{\dagger}+b\right)-E\right)\left|\Psi_{1}\right\rangle=0, \\
& \widetilde{\omega}\left|\Psi_{1}\right\rangle+\left(b^{\dagger} b-\lambda\left(b^{\dagger}+b\right)-E\right)\left|\Psi_{2}\right\rangle=0 .
\end{aligned}
$$

We now make the Bogoliubov transformation to the coherent bosons, $a^{\dagger}$ and $a$, specified by

$$
a^{\dagger}=b^{\dagger}-\lambda, \quad a=b-\lambda .
$$

The vacuum state of these bosons is the coherent state $|\lambda\rangle$. It should be noted that this choice of transformation may be intuited from considering the $\widetilde{\omega}=0$ limit of the Hamiltonian, where the same transformation is used to solve the model exactly in this limit, which is essentially equivalent to the displaced oscillator considered earlier. With this transformation Eq. (37) becomes

$$
\begin{gathered}
\widetilde{\omega}\left|\Psi_{2}\right\rangle+\left\{a^{\dagger} a+2 \lambda\left(a^{\dagger}+a\right)+3 \lambda^{2}-E\right\}\left|\Psi_{1}\right\rangle=0, \\
\widetilde{\omega}\left|\Psi_{1}\right\rangle+\left\{a^{\dagger} a-\lambda^{2}-E\right\}\left|\Psi_{2}\right\rangle=0,
\end{gathered}
$$

where the kets $\left|\Psi_{1,2}\right\rangle$ are now in the transformed representation. For these kets we choose the ansatz 


$$
\begin{gathered}
\left|\Psi_{1}\right\rangle=\sum_{n=0}^{N-1} p_{n}|n ; \lambda\rangle=\sum_{n=0}^{N-1} p_{n} \frac{\left(a^{\dagger}\right)^{n}}{\sqrt{n !}}|0 ; \lambda\rangle=P_{N-1}\left(a^{\dagger}\right)|0 ; \lambda\rangle, \\
\left|\Psi_{2}\right\rangle=\sum_{n=0}^{N} q_{n}|n ; \lambda\rangle=\sum_{n=0}^{N} q_{n} \frac{\left(a^{\dagger}\right)^{n}}{\sqrt{n !}}|0 ; \lambda\rangle=Q_{N}\left(a^{\dagger}\right)|0 ; \lambda\rangle,
\end{gathered}
$$

where $|n ; \lambda\rangle$ are number states of the coherent bosons, $a^{\dagger} a|n ; \lambda\rangle=n|n ; \lambda\rangle$, and we have introduced the polynomials $P_{N-1}$ and $Q_{N}$ of order $N-1$ and $N$, respectively. Making these substitutions we have

$$
\begin{aligned}
& \widetilde{\omega} \sum_{n=0}^{N} q_{n}|n ; \lambda\rangle+\sum_{n=0}^{N-1} p_{n}\left(n+3 \lambda^{2}-E\right)|n ; \lambda\rangle \\
& +2 \lambda \sum_{n=0}^{N-1} p_{n} \sqrt{n+1}|n+1 ; \lambda\rangle+2 \lambda \sum_{n=1}^{N-1} p_{n} \sqrt{n}|n-1 ; \lambda\rangle=0, \\
& \widetilde{\omega} \sum_{n=0}^{N-1} p_{n}|n ; \lambda\rangle+\sum_{n=0}^{N} q_{n}\left(n-\lambda^{2}-E\right)|n ; \lambda\rangle=0 .
\end{aligned}
$$

Considering the highest number state, $|N ; \lambda\rangle$, in the second of these equations, we see that for this equation to hold we require

$$
\left(N-\lambda^{2}-E\right) q_{N}=0 .
$$

Since $q_{N} \neq 0$ by ansatz, we obtain a determination of the energy

$$
E=N-\lambda^{2}
$$

This equation identifies the Juddian baseline energies, along which the Juddian solutions lie. Comparing the coefficients of the remaining number states gives us $2 N+1$ linear equations for the $2 N+1$ coefficients $\left(p_{m}, 0 \leqslant m \leqslant N-1\right)$ and $\left(q_{k}, 0 \leqslant k \leqslant N\right)$. To obtain nontrivial solutions, we clearly require the determinant of this equation set to be zero. This gives the compatibility condition, providing the locations of the Juddian points. The first two conditions $(N=1,2)$ have the explicit forms

$$
\begin{gathered}
\widetilde{\omega}^{2}+4 \lambda^{2}=1, \quad \text { for } \quad N=1, \\
\widetilde{\omega}^{4}+\left(12 \lambda^{2}-5\right) \widetilde{\omega}^{2}+32 \lambda^{4}-32 \lambda^{2}+4=0, \quad \text { for } \quad N=2,
\end{gathered}
$$

as have been given by Kuś and Lewenstein. ${ }^{9}$ Thus, for a given $N$, we have a polynomial of $N$ th order in $\lambda^{2}$ and $\widetilde{\omega}^{2}$. Each of these has $N$ roots for $\lambda^{2}$ in terms of $\widetilde{\omega}^{2}$, which all turn out to be real, thus giving the location of $N$ Juddian solutions. Before we look at these results, it is of interest to consider the other possible type of finite ansatz at the Juddian points. These are found by using the coherent bosons

$$
a^{\dagger}=b^{\dagger}+\lambda, \quad a=b+\lambda,
$$

and interchanging the roles of $\left|\Psi_{1}\right\rangle$ and $\left|\Psi_{2}\right\rangle$.

Results. By solving the complementary conditions we have calculated the first ten Juddian points for the resonant RH. These are displayed in Table I, listed to ten decimal places. 
TABLE I. The couplings, energies, and $N$, of the first ten Juddian points of the resonant Rabi Hamiltonian $\left(\omega=\omega_{0}=1\right)$.

\begin{tabular}{ccc}
\hline \hline$g$ & $E$ & $N$ \\
\hline 0.2165063510 & 0.8125000000 & 1 \\
0.1661640732 & 1.8895580031 & 2 \\
0.4460403578 & 1.2041919969 & 2 \\
0.1400889590 & 2.9215003343 & 3 \\
0.3664714887 & 2.4627945920 & 3 \\
0.6163829153 & 1.4802884071 & 3 \\
0.1234229399 & 3.9390671161 & 4 \\
0.3199075781 & 3.5906365658 & 4 \\
0.5243395120 & 2.9002723045 & 4 \\
0.7582492415 & 1.7002323511 & 4 \\
\hline \hline
\end{tabular}

The location of these Juddian points in the energy schema of the Hamiltonian is displayed in Fig. 1, where the schema was obtained by approximate numerical diagonalization via a standard configuration-interaction method, using a basis size of the lowest 101 harmonic oscillator states. ${ }^{14}$ Also plotted are the Juddian baselines from Eq. (43).

From Fig. 1 we see that the Juddian points occur at the level crossings in this diagram. Thus we see that they occur when two solutions of different parity $\Pi$ become degenerate in energy, and this degeneracy is the key to the existence of the Juddian solutions. The coherent-boson number states $|n ; \lambda\rangle$ are not eigenstates of $\Pi$, and thus the ansätze (40) is not of definite parity. It is precisely because we can construct wave functions of mixed parity that allows us to find such simple ansatz at the Juddian points.

We are now able to make explicit the connection between this method and that used by Kus and Lewenstein ${ }^{9}$ in investigating the $\mathrm{RH}$. They worked in the Bargmann representation, ${ }^{15}$ in which the bosonic operators are represented by

$$
b^{\dagger} \rightarrow z ; \quad b \rightarrow \frac{d}{d z}
$$

and postulated the following forms for the two components of the wave function:

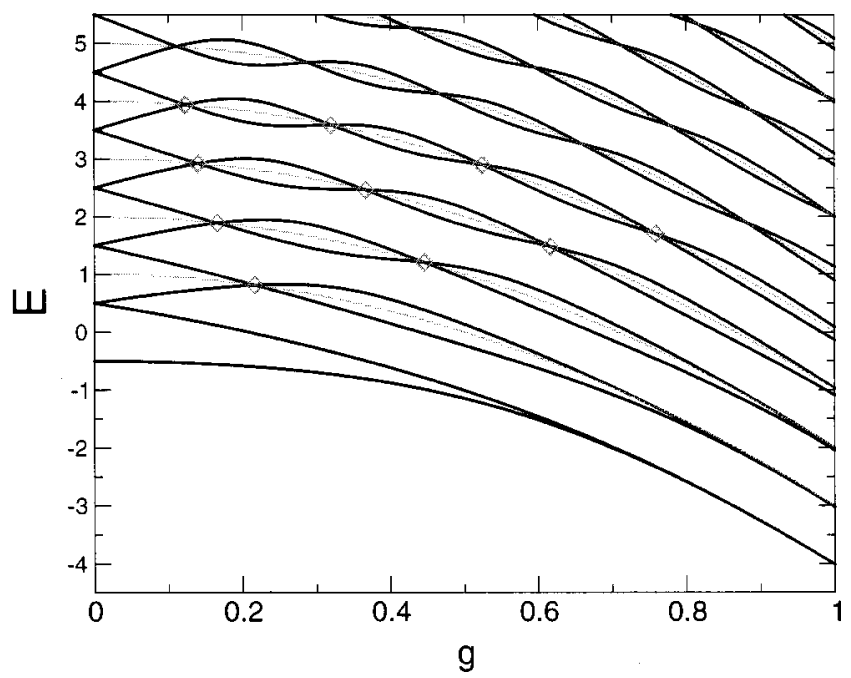

FIG. 1. The first ten Juddian points of the Rabi Hamiltonian (diamonds). Also plotted are the energy levels obtained by numerical diagonalization (dark lines), and the Juddian base lines (light lines). The Hamiltonian is resonant; $\omega=\omega_{0}=1$. 


$$
\begin{gathered}
\Psi_{1}(z)=e^{-|z|^{2} / 2}\left\langle z \mid \Psi_{1}\right\rangle=\widetilde{P}_{N-1}(z) e^{\lambda z}, \\
\Psi_{2}(z)=e^{-|z|^{2} / 2}\left\langle z \mid \Psi_{2}\right\rangle=\widetilde{Q}_{N}(z) e^{\lambda z},
\end{gathered}
$$

where $\widetilde{P}_{N-1}(z)$ and $\widetilde{Q}_{N}(z)$ are polynomials in $z$ of order $N-1$ and $N$, respectively. Bearing in mind the form of the coherent state (6), these wave functions are simply seen to be of the form of polynomials in the bosonic creation operator, $b^{\dagger}$, acting upon a coherent state of amplitude $\lambda$. In our ansatz (40), we have the same coherent state but now being acted upon by polynomials in $a^{\dagger}=\left(b^{\dagger}-\lambda\right)$, which shares a closer connection to the coherent state than $b^{\dagger}$.

The polynomials of Kuś and Lewenstein are simply related to those of ansatz (40) by $\widetilde{P}_{N}(z)=P_{N}(z-\lambda)$. In the present case where we have only used displacements of the boson mode, the difference between the two approaches is thus minimal. However, this is not the case when we require the use of squeezed bosons. Generally, an ansatz posited in the squeezed representation would contain polynomials of the form $P_{N}\left(c^{\dagger}\right)$, where $c^{\dagger}$ is the creation operator of the squeezed bosons. The analogous ansatz to Eq. (48) would still contain a polynomial in $z, \widetilde{P}_{N}(z)$ say. If we assume the simplest type of squeezing and write $c^{\dagger}=\left(b^{\dagger}+\sigma b\right) /\left(\sqrt{1-\sigma^{2}}\right)$ as in Eq. (25), then the Kuś and Lewenstein polynomial can be written

$$
\widetilde{P}_{N}(z)=P_{N}\left(\frac{z+\sigma \frac{d}{d z}}{\sqrt{1-\sigma^{2}}}\right),
$$

which, crucially, contains both $z$ and its derivative, and although formal relationships do exist between the polynomials of the two methods, these relationships are generally not trivial, especially if one considers the more general form of the Bogoliubov transformation. So the ansätze of the two methods are seen to be significantly different, and we conjecture that the one described here has several advantages which we shall discuss in the conclusion.

\section{CONCLUSIONS}

We have presented a method for finding isolated exact solutions of a class of nonadiabatic models, of the type frequently used in quantum optics and related fields.

Compared with the original approaches of Judd and Reik, the above-mentioned method is more transparent and considerably simpler, advantages that it shares with the technique of Kuś and Lewenstein. However, we believe that the use of transformed bosons is more obviously physically meaningful than the use of wave functions in Bargmann space, especially given the connection of these bosons to the coherent and squeezed states, so important in quantum optics.

As an example of the use of this technique, we have applied it to the Rabi Hamiltonian and obtained in a simple fashion the known Juddian solutions of this model. In this example, we have used the coherent bosons to obtain Juddian solutions for a problem with an interaction of the type $\lambda\left(b^{\dagger}+b\right) \sigma_{x}$. It is hopefully now clear how one may apply this method to further problems containing the same type of interaction. We have not as yet mentioned the application of the squeezed bosons in performing this kind of calculation. This second type of Bogoliubov transformation is useful in finding Juddian solutions of models containing two-photon type interactions. An obvious example is the two-photon Rabi Hamiltonian, ${ }^{16}$ which has the Hamiltonian

$$
H=\widetilde{\omega} \sigma_{z}+b^{\dagger} b+\lambda\left(b^{\dagger 2}+b^{2}\right) \sigma_{x} .
$$

Using squeezed bosons we are able to obtain a set of Juddian solutions for this model and these results will be discussed in a future publication.

Due to the intuitive nature and simplicity of this technique it is easy to extend to other systems. For example, in view of their mode of construction we expect that our displaced and squeezed coherent states will be of particular use in any quantum field theory that has underlying 
dynamical symmetry of the Weyl group or the $\mathrm{SU}(1,1)$ group, or to which the (inhomogeneous or homogeneous) Bogoliubov transformation may be profitably applied. The obvious grouptheoretical foundations of the technique also point the way to other approximations, since, for example in the squeezed (two-photon) case, $\mathrm{SU}(1,1)$ is not the only relevant group. Thus, the three-dimensional Lorentz group $\mathrm{SO}(2,1)$, which is the group of rotations in three-dimensional Minkowski space with two space and one time dimensions, is locally isomorphic to $\mathrm{SU}(1,1)$. Similarly, both the groups $\mathrm{SL}(2, \mathrm{R})$ of real second-order matrices with unit determinant and the symplectic group $\mathrm{Sp}(2, \mathrm{R})$ are also locally isomorphic to $\mathrm{SU}(1,1)$.

One may also readily generalize the current approach for the two-level models involving linear or quadratic interactions with a single boson (or canonical quantum mode) to the corresponding case of linear or bilinear interactions involving several distinct bosons or modes. For the linear models involving only displacements this is essentially trivial. However, for models involving squeezing, in the case of $n$ bosons or modes the various bilinear products of operators $b_{i}^{\dagger} b_{j}^{\dagger}$, $b_{i} b_{j}$ and $b_{i}^{\dagger} b_{j}, i, j=1,2, \ldots, n$ now form a realization of the higher symplectic algebra $\operatorname{Sp}(2 n, \mathbb{R})$. As before one can simply construct a unitary representation of this group by exponentiating the skew-adjoint operators in the algebra. For example, Bishop and Vourdas ${ }^{17}$ have shown explicitly how to construct the most general two-mode squeezed states associated with a unitary representation of the group $\mathrm{Sp}(4, \mathbb{R})$. Once again such states are the ordinary coherent states with respect to the new destruction operators $c_{1}$ and $c_{2}$, which are themselves general linear Bogoliubov transformations of the original destruction operators $b_{1}, b_{2}$ and their Hermitian-conjugate creation operators $b_{1}^{\dagger}, b_{2}^{\dagger}$. The $\mathrm{Sp}(4, \mathrm{R})$ algebra has various subalgebras corresponding to different sorts of linear pairing terms. For example, whereas the single-mode paring operators $K_{+}^{(i)} \equiv \frac{1}{2}\left(b_{i}^{\dagger}\right)^{2} ; K_{-}^{(i)}$ $\equiv \frac{1}{2} b_{i}^{2} ; K_{0}^{(i)} \equiv \frac{1}{2} b_{i}^{\dagger} b_{i}+\frac{1}{4}$ for $i=1,2$ correspond to the so-called $\left(\frac{1}{4}, \frac{3}{4}\right)$ representations of SU $(1,1)$, the mixed pairing operators $L_{+} \equiv b_{1}^{\dagger} b_{2}^{\dagger} ; L_{-} \equiv b_{1} b_{2} ; L_{0} \equiv \frac{1}{2}\left(b_{1}^{\dagger} b_{1}+b_{2}^{\dagger} b_{2}+1\right)$ correspond to the discrete-series representation of SU $(1,1)$. By contrast, the mixed pairing operators $J_{+} \equiv b_{1}^{\dagger} b_{2}$; $J_{-} \equiv b_{1} b_{2}^{\dagger} ; J_{0} \equiv \frac{1}{2}\left(b_{1}^{\dagger} b_{1}-b_{2}^{\dagger} b_{2}\right)$ correspond to the (Schwinger representation of) the angular momentum subalgebra $\mathrm{SU}(2)$. Bishop and Vourdas have shown in a separate publication ${ }^{18}$ how squeezed (pair) coherent states can also be used in connection with a rather broad class of quantum Lagrangians which include the damped harmonic oscillator, and hence with problems involving "quantum friction" or fluctuation-dissipation phenomena in general. Within quantum optics for example, the quantum theory of lasers and photon detection provide obvious applications. Such problems can now also usefully be extended by our present treatment to the case of such damped systems coupled to two level atoms.

The possibility of using these solutions as the basis of a perturbative approach extends the method away from just the isolated exact points to the remainder of the spectrum of the system. The properties of such an approach are yet to be investigated. Finally we note that the extension to similar single-mode or multimode systems as considered earlier coupled to $n$-level atoms with $n \geqslant 2$ is also straightforward in principle.

\section{ACKNOWLEDGMENT}

C.E. acknowledges the financial support of a research studentship from the Engineering and Physical Sciences Research Council (E.P.S.R.C.) of Great Britain.

\footnotetext{
${ }^{1}$ B. R. Judd, J. Chem. Phys. 67, 1174 (1977); J. Phys. C 12, 1685 (1979).

${ }^{2}$ L. Allen and J. H. Eberly, Optical Resonance and Two-Level Atoms (Wiley, New York, 1975).

${ }^{3}$ H. G. Reik, H. Nusser, and L. A. Amarante Ribeiro, J. Phys. A 15, 3491 (1982); M. Kuś, J. Math. Phys. 26, 2792 (1985); H. G. Reik and M. Doucha, Phys. Rev. Lett. 57, 787 (1986); H. G. Reik, P. Lais, M. E. Stützle, and M. Doucha, J. Phys. A 20, 6327 (1987).

${ }_{4}^{4}$ I. D. Feranchuk, L. I. Komarov, and A. P. Ulyanenkov, J. Phys. A 29, 4035 (1996).

${ }^{5}$ R. F. Bishop, N. J. Davidson, R. M. Quick, and D. M. van der Walt, Phys. Rev. A 54, 4657 (1996).

${ }^{6}$ R. F. Bishop, N. J. Davidson, R. M. Quick, and D. M. van der Walt, Phys. Lett. A 254, 215 (1999).

${ }^{7}$ R. Graham and M. Höhnerbach, Phys. Lett. A 101, 61 (1984).

${ }^{8}$ G. Hose and H. S. Taylor, Phys. Rev. Lett. 51, 947 (1983).

${ }^{9}$ M. Kuś, J. Math. Phys. 26, 2792 (1985); M. Kuś and M. Lewenstein, J. Phys. A 19, 305 (1986).

${ }^{10}$ R. R. Puri and R. K. Bullough, J. Opt. Soc. Am. B 5, 2021 (1988).
} 
${ }^{11}$ R. Glauber, Phys. Rev. 131, 2766 (1963).

${ }^{12}$ R. F. Bishop and A. Vourdas, J. Phys. A 19, 2525 (1986).

${ }^{13}$ A. Perelomov, Generalised Coherent States and Their Applications (Springer, Berlin, 1986).

${ }^{14}$ C. Emary, Ph.D. thesis, UMIST, Manchester, 2001.

${ }^{15}$ V. Bargmann, Commun. Pure Appl. Math. 14, 187 (1964).

${ }^{16}$ K. M. Ng, C. F. Lo, and K. L. Liu, Eur. Phys. J. D 6, 119 (1999); C. F. Lo, K. L. Liu, and K. M. Ng, Europhys. Lett. 42 , 1 (1998).

${ }^{17}$ R. F. Bishop and A. Vourdas, Z. Phys. B: Condens. Matter 17, 527 (1988).

${ }^{18}$ R. F. Bishop and A. Vourdas, J. Phys. A 20, 3727 (1987). 